\title{
Obtención de silicatos de calcio utilizando el método de precipitación controlada
}

\author{
J. E. RODRÍGUEZ-PÁEZ', L. M. AHUMADA², J. M. BUSTAMANTE , J. RUIZ DE MURGUEITIO ${ }^{3}$ \\ ${ }^{1}$ Grupo CYTEMAC. Departamento de Física - FACNED \\ ${ }^{2}$ Departamento de Química - FACNED \\ ${ }^{3}$ Departamento de Geotecnia - FIC \\ Universidad del Cauca. Calle 5 № 4 - 70 Popayán - Cauca/ COLOMBIA
}

\begin{abstract}
Los silicatos de calcio hidratados son compuestos del sistema $\mathrm{SiO}_{2}-\mathrm{CaO}-\mathrm{H}_{2} \mathrm{O}$. Estos materiales se utilizan para fabricar ladrillos de cal-arena, hormigones aireados (espumados) u "hormigón celular", ladrillos de cal-flint, como material de relleno en las industrias de productos de caucho, pinturas y plásticos, entre otros posibles usos. Indudablemente el grupo de las tobermoritas es el más interesante debido a su papel importante en la hidratación del cemento: los silicatos tricálcico (3CaO. $\left.\mathrm{SiO}_{2}-\mathrm{C}_{3} \mathrm{~S}\right)$ y dicálcico $\left(2 \mathrm{CaO}, \mathrm{SiO}_{2}-\mathrm{C}_{2} \mathrm{~S}\right)$ son los principales constituyentes del cemento Pórtland. En este trabajo se muestra la obtención de compuestos de silicato de calcio a través del método de precipitación controlada usando como fuente de silicio el dióxido de silicio, $\mathrm{SiO}_{2}$ obtenido de la cascarilla de arroz. Se analizan los fenómenos fisicoquímicos que ocurren durante el proceso de síntesis y se estudia la evolución de las fases cristalinas del producto obtenido, al ser sometido a tratamientos térmicos, utilizando para ello Difracción de Rayos X (DRX) y espectroscopía infrarroja (FTIR).
\end{abstract}

Palabras clave: Silicatos de calcio; precipitación controlada; cascarilla de arroz.

Synthesis of hydrated calcium silicate using the controlled precipitation method

Calcium silicate hydrates are compounds of $\mathrm{SiO}_{2}-\mathrm{CaO}-\mathrm{H}_{2} \mathrm{O}$ system. These materials are used to make lime-sand bricks concretes, or "cellular concretes", lime-flint brices and as fillers in rubber, paint and plastic products industries, among others. Undoubtedly, the tobermoritas is the most interesting group given its important role in concrete hydration: hydrated calcium silicate $(\mathrm{C}-\mathrm{S}-\mathrm{H})$ is the main concrete hydration product and its primary bounding phase. The hydrothermal method is the most frequently used in the synthesis of these materials. This work shows the synthesis of hydrated calcium silicates through the controlled precipitation method using silicon dioxide $\mathrm{SiO}_{2}$, as raw material obtained from rice husk. The physicochemical phenomena that occur during the calcium silicates synthesis process were analized. The crystalline phases of the final product when exposed to thermic treatments were studied as well using $\mathrm{x}$-ray diffraction and Infrared spectroscopy.

Keywords: Calcium silicate hydrates; controlled precipitation: rice husk ash.

\section{INTRODUCCIÓN}

Los silicatos de calcio hidratados son compuestos del sistema $\mathrm{SiO}_{2}-\mathrm{CaO}-\mathrm{H}_{2} \mathrm{O}$ (1). El incremento del conocimiento sobre estos compuestos, y la comprensión de cómo influyen las condiciones de síntesis sobre sus propiedades, han abierto nuevas posibilidades de utilización de estos compuestos más allá de la fabricación de elementos de construcción (ladrillos de cal-arena, ladrillos de cal-flint, hormigones aireados o espumados, placas prefabricadas, etc.) y de utilizarlos como material de relleno en industrias de productos de caucho, pinturas y plásticos (1).

El método hidrotermal es el que más se utiliza en la obtención de silicatos de calcio hidratados (2). Concretamente para la obtención de las tobermoritas (1), de particular importancia en la hidratación del cemento (3), se han utilizado otros métodos de síntesis destacándose el de precursor polimérico o Pechiní (4-6). El efecto que la naturaleza de los precursores de calcio y silicio, sobre el tipo de silicato de calcio obtenido, no se ha estudiado a profundidad. Se han utilizado como precursores de calcio, principalmente, carbonato (7), nitrato (4) e hidróxido (8), y un sol de sílice o sílice coloidal $(4,7)$, como precursor de silicio. En el presente trabajo se utilizó $\mathrm{SiO}_{2}$ obtenido de la cascarilla de arroz como fuente de
Silicio. Diversos estudios se han realizado previamente sobre el uso de este $\mathrm{SiO}_{2}$ en la fabricación de cemento (8-10) pero muy poco se ha considerado su uso como precursor durante la síntesis de silicatos de calcio en general.

El método de síntesis que se utilizó en el presente trabajo fue el de precipitación controlada, método que ha sido empleado con éxito en la obtención de otros sistemas (11), que permite tener un control sobre las variables del proceso para garantizar la reproducibilidad del mismo y es uno de los métodos que presenta más posibilidad de implementación industrial. El producto cerámico obtenido se sometió a diferentes tratamientos térmicos y se realizo el seguimiento de la evolución de las fases cristalinas, como consecuencia del tratamiento, utilizando difracción de rayos $\mathrm{X}$ y la de los grupos funcionales empleando Espectroscopía Infrarroja.

\section{PARTE EXPERIMENTAL}

El $\mathrm{SiO}_{2}$ que se utilizó en el presente proyecto se obtuvo de la cascarilla de arroz a través del proceso que se describe en la literatura (12). El método de precipitación controlada (MPC) 
también ya ha sido descrito previamente (13). Se tomaron $4.814 \mathrm{~g} \mathrm{de} \mathrm{SiO}_{2}$, obtenido de la manera indicada anteriormente, y se dispersaron en una solución acuosa $0.1 \mathrm{M}$ de $\mathrm{HNO}_{3^{\prime}}$ agitando el sistema a 400 r.p.m. Posteriormente se adicionó a la solución $32.317 \mathrm{~g}$ de acetato de calcio, $\mathrm{Ca}\left(\mathrm{CH}_{3} \mathrm{COO}\right)_{2}$ (Analyticals), para conformar una solución de acetato con una concentración de $0.05 \mathrm{M}$ manteniendo el sistema en continua agitación. Se procedió a la adición de una base con el fin de favorecer la hidrólisis del $\mathrm{Ca}^{2+}$. Se utilizo tanto una base débil, hidróxido de amonio ( $\mathrm{NH}_{4} \mathrm{OH}$ - Mallinckrodt), como base fuerte, hidróxido de sodio ( $\mathrm{NaOH}-$ Mallinckrodt). El seguimiento de la variación del $\mathrm{pH}$ del sistema se realizó utilizando un pHmetro marca Metrohm 744. Se observó mayor formación de fase sólida en el seno de la solución cuando se utilizó $\mathrm{NaOH}$. Para evitar la incorporación en el sistema de una gran cantidad de $\mathrm{Na}^{+}$, se utilizó una solución diluida de la base $(0.5 \mathrm{M}$ de $\mathrm{NaOH})$. Se trabajó inicialmente a temperatura ambiente pero se observó que a temperaturas mayores se formaba más fase sólida en el seno del sistema, por lo cual el $\mathrm{NaOH}$ se adicionó a $60^{\circ} \mathrm{C}$ para que las reacciones de hidrólisis y condensación, y por lo tanto la formación del precipitado, se vea favorecida (forzar la hidrólisis).

Todos los sistemas estudiados se secaron a $70^{\circ} \mathrm{C}$ en una estufa, durante dos días, y luego se molieron con un mortero de ágata. El polvo cerámico obtenido se sometió a tratamientos térmicos a $500^{\circ} \mathrm{C}, 1000^{\circ} \mathrm{C}$ y $1100^{\circ} \mathrm{C}$ durante dos horas a una velocidad de calentamiento de $5^{\circ} \mathrm{C} / \mathrm{min}$. Estas fueron las muestras que se utilizaron para los estudios de DRX y FTIR. Los difractógramas de rayos $\mathrm{X}$ se tomaron utilizando un difractómetro Siemmens - D5000 con una fuente de $\mathrm{Cu}(\mathrm{K} \alpha)$ operando a $40 \mathrm{KV}$ y $40 \mathrm{~mA}$ y los espectros se registraron empleando un equipo ST Termo Nicolette.

\section{RESULTADOS Y DISCUSION}

El dióxido de silicio obtenido, siguiendo el proceso indicado en la literatura (12), es amorfo tal como lo muestra su difractograma de rayos $X$ (14). Los aglomerados del polvo cerámico tenían un tamaño entre 1 y $5 \mu \mathrm{m}$, con una alta distribución de tamaños y una morfología irregular. El valor de superficie especifica, determinado a través del método BET, fue de $230 \mathrm{~m}^{2} / \mathrm{g}(14)$.

En la figura 1 se muestran las curvas de valoración potenciométrica obtenidas para el sistema $\mathrm{SiO}_{2}-$
$\mathrm{Ca}\left(\mathrm{CH}_{3} \mathrm{COOH}\right)_{2}$, en una solución acuosa acidificada cuando se utilizó como precipitante $\mathrm{NH}_{4} \mathrm{OH}$, figura $1(\mathrm{a}), \mathrm{y} \mathrm{NaOH}$, figura 1(b). Con el fin de garantizar una producción efectiva de la fase sólida en el sistema acuoso acidificado de $\mathrm{SiO}_{2}-$ $\mathrm{Ca}\left(\mathrm{CH}_{3} \mathrm{COO}\right)_{2}$, y considerando que el $\mathrm{Ca}(\mathrm{OH})_{2}$ comienza a precipitar a $\mathrm{pH}>11(15)$, la solución se calentó a $60^{\circ} \mathrm{C}$ mientras se adicionaba la base fuerte, $\mathrm{NaOH}$. La figura 1(b) muestra el comportamiento del sistema durante el proceso. La curva de valoración potenciométrica obtenida, figura 1(b), presenta tres regiones bien definidas y las curvas de valoración que se obtuvieron al variar la concentración de precursor de calcio, y la cantidad de ácido presente en la solución, presentan un comportamiento muy similar. La figura 1(a) obtenida para el sistema precipitado con $\mathrm{NH}_{4} \mathrm{OH}$ no presenta la primera región.

En todos los sistemas de $\mathrm{SiO}_{2}-\mathrm{Ca}\left(\mathrm{CH}_{3} \mathrm{COO}\right)_{2}$ que se calentaron a $60^{\circ} \mathrm{C}$, durante la adición el $\mathrm{NaOH}$, fue evidente la existencia de una suspensión coloidal además de la presencia de la fase dispersa de $\mathrm{SiO}_{2}$ y a los dos días de envejecimiento se observó la formación de un gel. Algo diferente sucedió en el sistema precipitado con $\mathrm{NH}_{4} \mathrm{OH}$ donde se observó la formación de un sedimento.

\section{Hidrólisis del $\mathrm{Ca}^{2+}$}

$\mathrm{El} \mathrm{Ca}^{2+}$ es un catión poco ácido debido a su gran tamaño. Observando su diagrama del logaritmo de concentración en función del $\mathrm{pH}(16)$, el hidróxido de calcio $\mathrm{Ca}(\mathrm{OH})_{2}$ comienza a precipitar a un $\mathrm{pH}>11$ y debido a su elevado radio iónico tiene poca tendencia a formar complejos. La presencia de $\mathrm{NH}_{4}^{+}$ en exceso, ácido conjugado débil, es suficiente para aumentar notablemente la solubilidad e impedir la precipitación total. Esto último justifica la ineficiencia del $\mathrm{NH}_{4} \mathrm{OH}$ para generar una fase sólida en el seno de la solución (16), situación observada en uno de los sistemas estudiados.

Considerando la hidrólisis del calcio, se ha realizado un cuidadoso estudio de la asociación del ion hidroxilo con el ion calcio para producir el par iónico (17):

$$
\mathrm{Ca}^{2+}+\mathrm{OH} \Leftrightarrow \mathrm{CaOH}^{+}
$$

en un rango de temperatura entre $0^{\circ} \mathrm{C}$ y $40^{\circ} \mathrm{C}$. Esta formación de pares de iones $\mathrm{OH}-\mathrm{Ca}^{2+}$ debe aumentar la solubilidad en el sistema. De acuerdo a lo anterior, en el sistema acuoso en estudio, se deben tener como especies principales de calcio
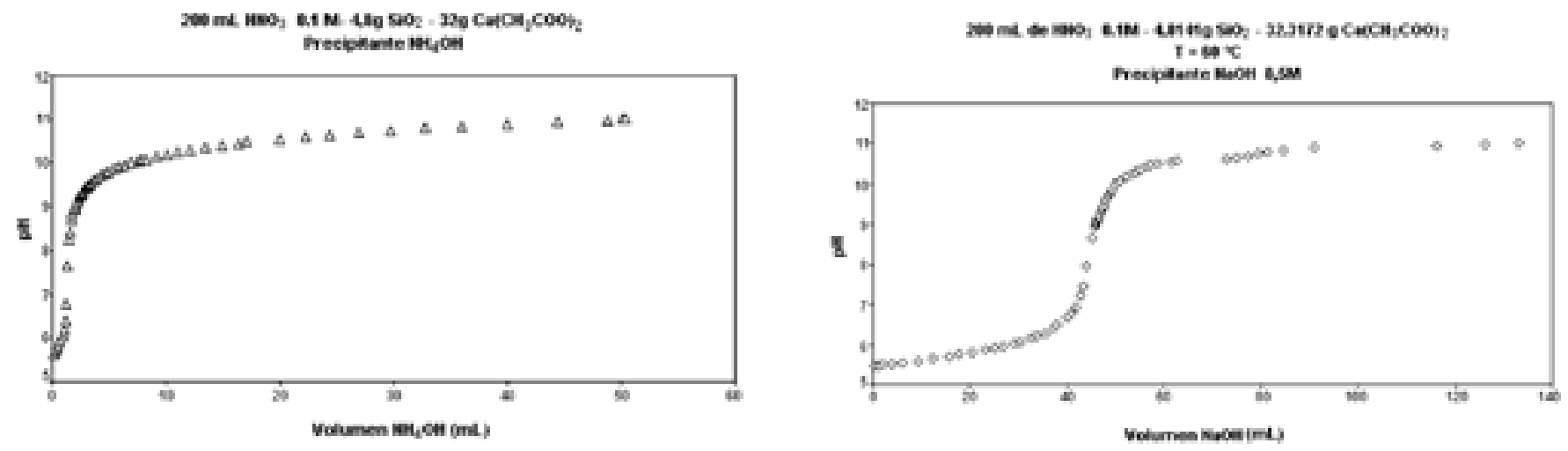

Fig. 1- Curvas de Valoración potenciométrica del sistema $\mathrm{SiO}_{2}-\mathrm{Ca}\left(\mathrm{CH}_{3} \mathrm{COO}\right)_{2}$ utilizando como precipitante (a) $\mathrm{NH}_{4} \mathrm{OH}$ y (b) $\mathrm{NaOH}$. 
el par iónico $\mathrm{CaOH}^{+}$y $\mathrm{Ca}(\mathrm{OH})_{2}$ para $\mathrm{pH}>11$. La formación de $\mathrm{Ca}(\mathrm{OH})_{2}$ debe producirse de acuerdo a la siguiente reacción para el caso de la base fuerte:

$\mathrm{Ca}\left(\mathrm{CH}_{3} \mathrm{COO}\right)_{2 \text { (асио) }}+\mathrm{Na}(\mathrm{OH})_{\text {(асио) }} \Leftrightarrow \mathrm{Ca}(\mathrm{OH})_{2 \text { (s) }}+\mathrm{NaCH}_{3} \mathrm{COO}_{\text {(асио) }}$

Como indica la teoría clásica de Bjerrum (18), debe existir una distancia crítica $\mathrm{r}^{*}$ por debajo de la cual la probabilidad de la asociación de iones se incrementa rápidamente; para el sistema en estudio se tendría un valor de $\mathrm{r}^{*} \sim 0.71 \mathrm{~nm}$. Sin embargo, en soluciones acuosas, es de esperar que esta distancia de separación no sea la más frecuente y que por lo tanto el $\mathrm{Ca}^{2+}$ esté solvatado (numero de hidratación 6) (19). Esto justifica la difícil formación de $\mathrm{Ca}(\mathrm{OH})_{2}$ y de que el $\mathrm{Ca}^{2+}$ permanezca disuelto en prácticamente todo el rango de $\mathrm{pH}$ de trabajo (20).

Al adicionar el $\mathrm{HNO}_{3}$ debe disminuir la constante dieléctrica del sistema afectandola estructura interna del agua. La literatura reporta (18) que en solventes con constante dieléctrica baja la formación de pares de iones es sustancialmente alta. Los pares iónico se pueden asociar en una variedad de agregados tales como pares de pares y aglomerados mayores (21). El inicio de la polimerización, del gel que se observa en algunos sistemas estudiados, se puede dar a través de la mediación de pares de iones, proceso bastante complejo, o por la aglomeración de los pares de iones en grandes asociaciones como se ha observado en otros sistemas: poliesteril litio y polidientil litio (21).

\section{Caracterización de la fase sólida.}

Se utilizó espectroscopia infrarroja para determinar los grupos funcionales presentes en las muestras obtenidas utilizando $\mathrm{NH}_{4} \mathrm{OH}$ sin ácido, $\mathrm{A} 1$, y con ácido, $\mathrm{A} 2$, y el $\mathrm{NaOH}$ sin ácido, A3, y con ácido, A4; los espectros se muestran en la figura 2.

Los resultados más destacados de este análisis se indican a continuación. Comparando los espectros de los sistemas sin ácido y diferente precipitante (A3 y A1), figuras 2 (a) y (b), se observa una banda centrada en $\sim 3450 \mathrm{~cm}^{-1}$ que es más ancha para la muestra precipitada con $\mathrm{NH}_{4} \mathrm{OH}$ debido a la presencia de "aminas", provenientes del hidróxido de amonio, adicional a los $\mathrm{OH}$ asociados al agua. Además, las bandas entre 1400 - $1700 \mathrm{~cm}^{-1}$ están más definidas en la muestra A3 precipitada con $\mathrm{NaOH}$. Por otro lado, en el espectro de la muestra A1, es evidente una banda a $960 \mathrm{~cm}^{-1}$ la cual puede corresponder a un silicato de calcio intermedio entre el $\mathrm{C}_{3} \mathrm{~S}, 940 \mathrm{~cm}^{-1}$, y el $\beta-C_{2} S$, $999 \mathrm{~cm}^{-1}$. Finalmente también aparecen bandas a 880 y 854 $\mathrm{cm}^{-1}$, para la muestra A1, donde la banda a $880 \mathrm{~cm}^{-1}$ se puede asociar a un enlace que involucra al calcio.

Para los sistemas con ácido y diferente precipitante, figuras 2 (c) y (d), se observa en el espectro de la muestra A4 una banda a $3800 \mathrm{~cm}^{-1}$ que indica la presencia de grupos silanoles $\mathrm{Si}-\mathrm{OH}$ [21]. En el espectro de la muestra A2 aparecen dos bandas a $2927 \mathrm{~cm}^{-1}$ y $2857 \mathrm{~cm}^{-1}$ que corresponden al $v$ $(\mathrm{C}-\mathrm{H})$ y al $v_{\mathrm{s}}(\mathrm{C}-\mathrm{H})$, respectivamente. En la región entre 1300 $-1700 \mathrm{~cm}^{-1}$ se presenta una gran variación entre los espectros, siendo mayor la cantidad de bandas presentes en el espectro correspondiente a la muestra A4 que en el de la A2.

Otro cambio apreciable está relacionado con la banda a $1100 \mathrm{~cm}^{-1}$. Mientras en el espectro de la muestra A2 están muy bien definidas las bandas del $\mathrm{SiO}_{2}\left(1100 \mathrm{~cm}^{-1}, 803 \mathrm{~cm}^{-1}\right.$ y $471 \mathrm{~cm}^{-1}$ ), en la muestra A4 no ocurre lo mismo, en ella

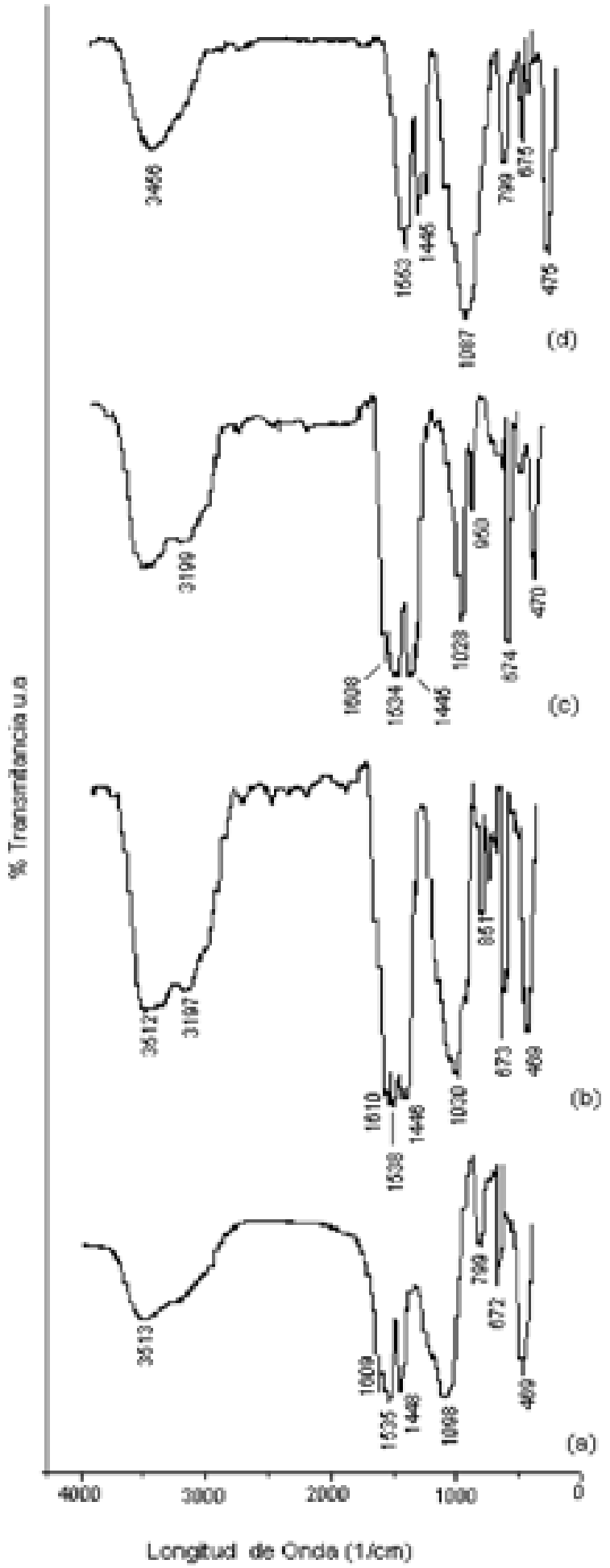

Fig. 2- Espectros IR correspondientes a muestras, precipitadas con hidróxido de sodio (a) A3, (c) A4, y con hidróxido de amonio: (b) A1 y (d) A2.

aparece una banda a $1050 \mathrm{~cm}^{-1}$, asociada al $\mathrm{NO}_{3}^{-}$, otra a $1028 \mathrm{~cm}$ ${ }_{1}^{1}$ que corresponde al $\delta\left(\mathrm{CH}_{3}\right)$, un hombro a $800 \mathrm{~cm}^{-1}$ y una banda a $470 \mathrm{~cm}^{-1}$ que estaría asociada a un modo vibracional del $\mathrm{SiO}_{2}$ [22]. En la muestra A2 se observa claramente la presencia del $\mathrm{SiO}_{2}$, utilizado como precursor. De acuerdo con 


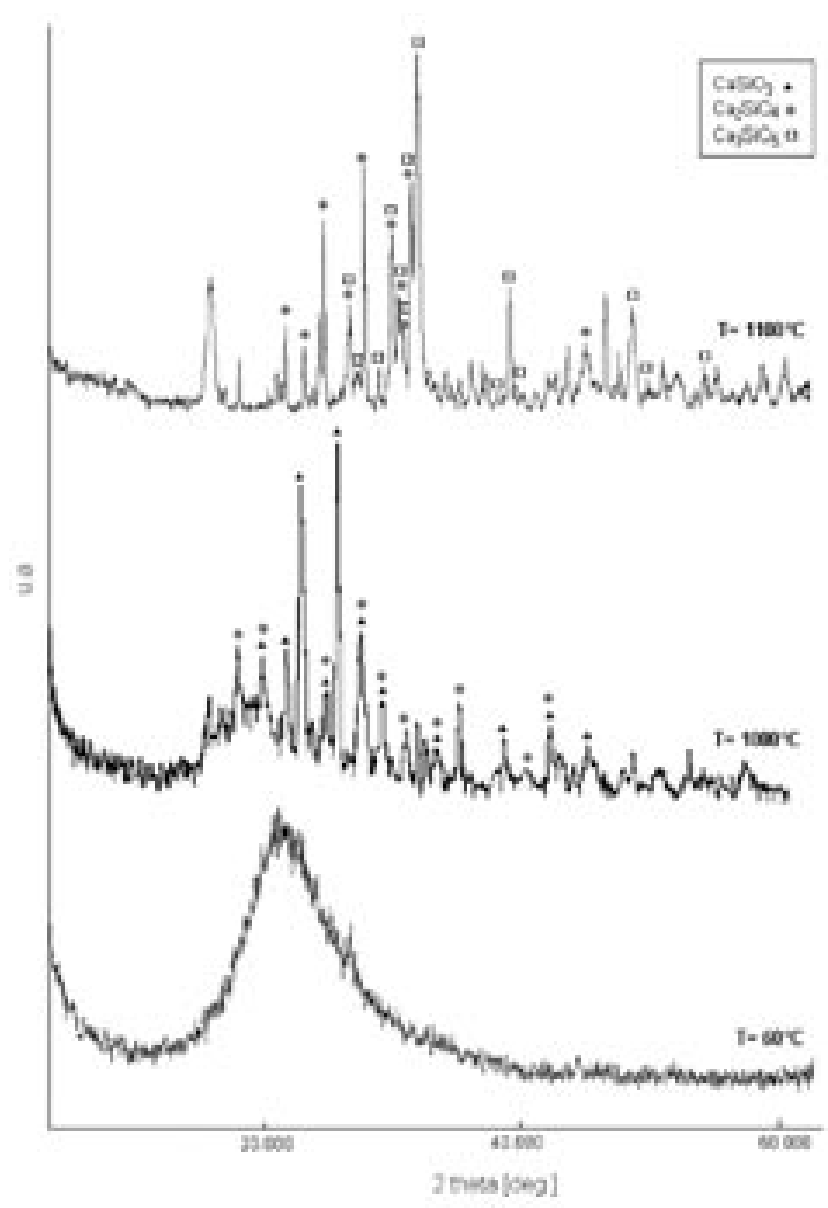

Fig. 3- Difractogramas de rayos $\mathrm{X}$ de muestras sólidas correspondientes al sistema $\mathrm{SiO}_{2}-\mathrm{Ca}\left(\mathrm{CH}_{3} \mathrm{COO}\right)_{2}$, precipitadas con $\mathrm{NH}_{4} \mathrm{OH}$ y tratadas térmicamente a: (a) secado $60^{\circ} \mathrm{C}$, (b) $1000^{\circ} \mathrm{C}$ y (c) $1100^{\circ} \mathrm{C}$.

las características del espectro, la muestra A4 (figura 2 (c)) experimenta un mayor avance en las reacciones entre $\mathrm{el} \mathrm{SiO}_{2} \mathrm{y}$ el compuesto de calcio.

Para determinar las fases cristalinas presentes en el sólido que se conforma es los sistemas, se utilizo difracción de rayos
$X$. La figura 3 ilustra los difractogramas de rayo $\mathrm{X}$ de la muestra obtenida después de la adición del $\mathrm{NH}_{4} \mathrm{OH}$ y cuando ésta es sometida a diversos tratamientos térmicos. El resumen de la evolución de fases de esta muestra (denominada A1), al ser tratada térmicamente a $1000^{\circ} \mathrm{C}$ y $1100^{\circ} \mathrm{C}$, se ilustra en la tabla 1.

El efecto de la variación de la naturaleza del precipitante, y de la presencia del $\mathrm{HNO}_{3}$ en el sistema, sobre las fases cristalinas que existen en las muestras sólidas analizadas, se indica en la figura 4. Además se presentan los difractogramas cuando las anteriores muestras se trataron térmicamente. El resumen de la evolución de las fases para estas muestras sólidas (A2 sin ácido y A3 con ácido), al ser sometidas a tratamientos térmicos, se indica en la tabla 1.

Un efecto interesante se presenta cuando la muestra a temperatura ambiente es amorfa (muestra A1 - figura 3). El difractógrama correspondiente a la muestra secada a $60^{\circ} \mathrm{C}$ coincide con el del $\mathrm{SiO}_{2}$ amorfo. Al tratarla térmicamente se van conformando los silicatos de calcio tal como se ilustra en la tabla 1. Esto lleva a considerar que, durante la adición del $\mathrm{NH}_{4} \mathrm{OH}$, las partículas de $\mathrm{SiO}_{2}$ inicialmente son recubiertas por especies químicas de calcio y que al tratar térmicamente el sistema se favorece la difusión de este catión hacia el interior de las mismas, ocasionando la reacción entre el $\mathrm{SiO}_{2}$ y el calcio y la producción de los silicatos de calcio correspondientes.

\section{CONCLUSIONES}

Considerando los resultados obtenidos en el presente trabajo se puede concluir lo siguiente:

1- Las curvas de valoración potenciométrica obtenidas permiten controlar el proceso y garantizar la reproducibilidad del método; además ellas contienen información sobre la evolución de los diferentes procesos fisicoquímicos que ocurren durante el proceso. Las especies químicas del calcio que predominan en el sistema son pares iónicos $\mathrm{CaOH}^{+}$, agregados de pares de pares y aglomerados mayores. La formación de los pares iónicos se ve favorecida por la disminución de la constante dieléctrica del sistema debido a la adición del ácido.

TABLA I. FASES CRISTALINAS DE LOS SILICATOS DE CALCIO QUE SE FORMAN DURANTE EL TRATAMIENTO TÉRMICO A QUE ES SOMETIDA LA MUESTRA SÓLIDA OBTENIDA DEL SISTEMA $\mathrm{SIO}_{2}-\mathrm{CA}\left(\mathrm{CH}_{3} \mathrm{COO}\right)_{2}$ UTILIZANDO DIFERENTES PRECIPITANTES Y CONDICIONES PARA EL PROCESO DE SÍNTESIS.

\begin{tabular}{|c|c|c|c|}
\hline MUESTRA & $\begin{array}{c}\text { TEMPERATURA } \\
\text { AMBIENTE }\end{array}$ & $\mathrm{T}=1000^{\circ} \mathrm{C}$ & $\mathrm{T}=1100^{\circ} \mathrm{C}$ \\
\hline A1 & Amorfa & $\begin{array}{l}\mathrm{CaSiO}_{3}-(\mathrm{PDF} 420550) \\
\mathrm{Ca}_{2} \mathrm{SiO}_{4}-(\mathrm{PDF} 231045)\end{array}$ & $\begin{array}{l}\mathrm{Ca}_{2} \mathrm{SiO}_{4}-(\mathrm{PDF} 360642) \\
\mathrm{Ca}_{3} \mathrm{SiO}_{5}-(\mathrm{PDF} 420551)\end{array}$ \\
\hline A2 & $\begin{array}{c}\mathrm{CaSiO}_{3}-(\mathrm{PDF} 450156) \\
\mathrm{Ca}_{2} \mathrm{SiO}_{4}-(\mathrm{PDF} 231045) \\
\mathrm{Ca}_{8} \mathrm{Si}_{5} \mathrm{O}_{18}-(\mathrm{PDF} 290368)\end{array}$ & $\begin{array}{l}\mathrm{CaSiO}_{3}-(\mathrm{PDF} 450156) \\
\mathrm{Ca}_{2} \mathrm{SiO}_{4}-(\mathrm{PDF} 360642)\end{array}$ & $\begin{array}{c}\mathrm{CaSiO}_{3}-(\mathrm{PDF} 450156) \\
\mathrm{Ca}_{2} \mathrm{SiO}_{4}-(\mathrm{PDF} 360642)\end{array}$ \\
\hline A3 & $\begin{array}{l}\mathrm{CaSiO}_{3}-(\mathrm{PDF} 180306) \\
\mathrm{Ca}_{2} \mathrm{SiO}_{4}-(\mathrm{PDF} 200337)\end{array}$ & $\begin{array}{l}\mathrm{CaSiO}_{3}-(\mathrm{PDF} 420550) \\
\mathrm{CaSiO}_{3}-(\mathrm{PDF} 270088) \\
\mathrm{Ca}_{2} \mathrm{SiO}_{4}-(\mathrm{PDF} 360642)\end{array}$ & $\begin{array}{l}\mathrm{CaSiO}_{3}-(\mathrm{PDF} 420550) \\
\mathrm{CaSiO}_{3}-(\mathrm{PDF} 270088)\end{array}$ \\
\hline
\end{tabular}



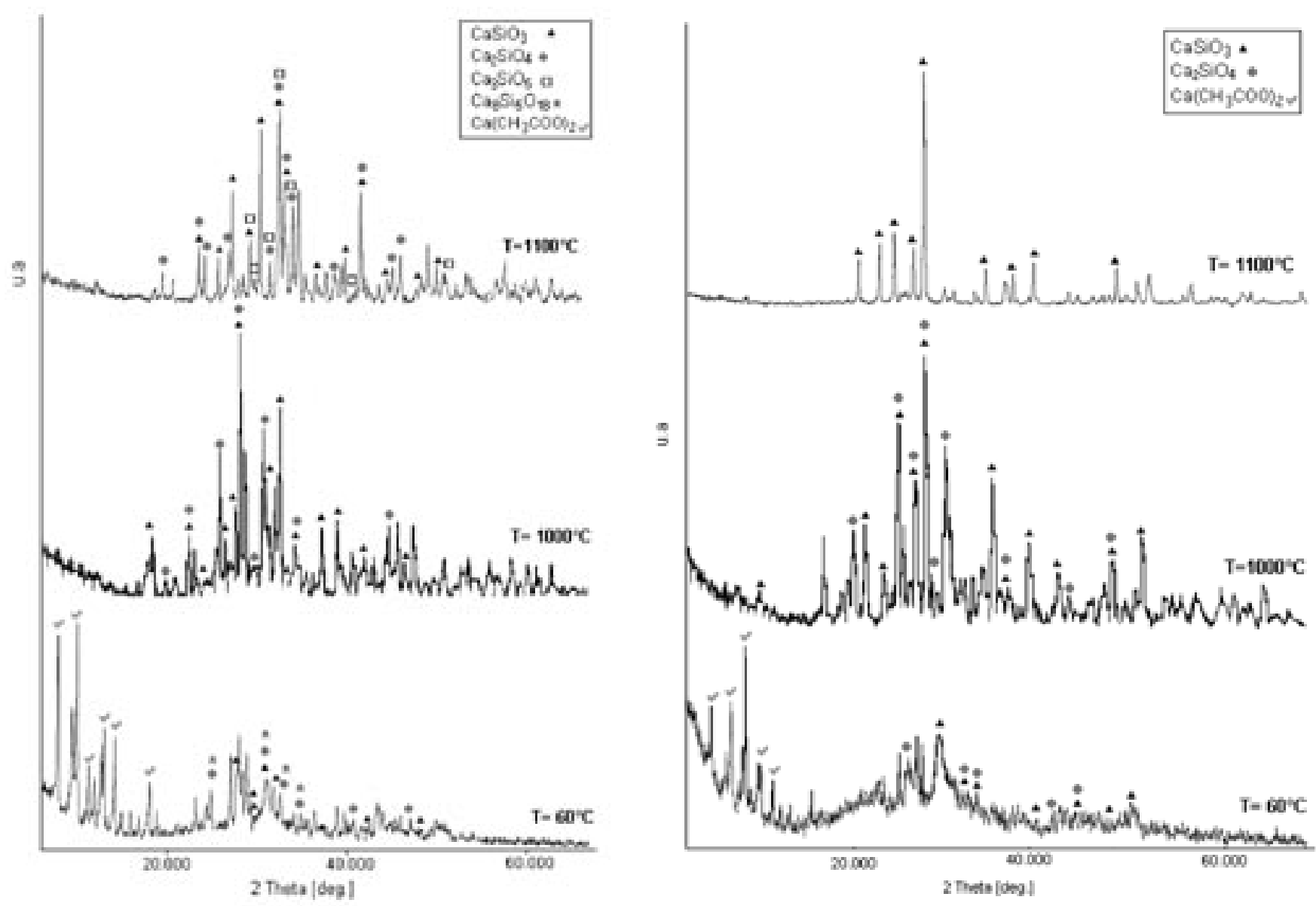

Fig.4- Difractogramas de rayos $\mathrm{X}$ de muestras sólidas correspondientes al sistema $\mathrm{SiO}_{2}-\mathrm{Ca}\left(\mathrm{CH}_{3} \mathrm{COO}\right)_{2}$, obtenidas en (a) solución acuosa y (b) solución acidificada $0.1 \mathrm{~N} \mathrm{HNO}_{3}$ y que fueron sometidas a diferentes tratamientos térmicos ( secada a $60^{\circ} \mathrm{C}$ y tratadas a $1000^{\circ} \mathrm{C}$ y $1100^{\circ} \mathrm{C}$ )

2- En todos los sistemas $\mathrm{SiO}_{2}-\mathrm{Ca}\left(\mathrm{CH}_{3} \mathrm{COO}\right)_{2}$ estudiados, con condiciones de síntesis diferentes, se obtuvieron silicatos de calcio pero a los que se les adicionó $\mathrm{NH}_{4} \mathrm{OH}$ no se forma fase sólida debido a la existencia de exceso de $\mathrm{NH}_{4}^{+}$lo que aumenta notablemente la solubilidad de las especies químicas de calcio presentes en el sistema. Especies químicas de calcio se deben adsorber sobre la superficie de las partículas de $\mathrm{SiO}_{2}$ y difunden a través de ellas cuando se activan térmicamente, favoreciendo las reacciones químicas y la formación de compuestos de silicato de calcio, entre ellos los polimorfos de $\mathrm{C}_{2} \mathrm{~S}$ y $\mathrm{C}_{3} \mathrm{~S}$. De los resultados de DRX se puede concluir que la reactividad química del sistema es bastante elevada y que las condiciones de síntesis determinan la naturaleza del producto final.

\section{REFERENCIAS}

1. H.F.W. Taylor, Cement chemistry, 2nd ed. Thomas Telford, London, U.K., 1997. 2. A. R. West, Solid State Chemistry and its applications, John Wiley \& Sons Inc, 1984.

3. I. Odler, Special inorganic cements, Modern Concrete Technology 8, E\&FN, Taylor \& Francis Group, 2000.

4. S. Hong, J.F. Young, "Hydration and Phase Stability of dicalcium silicate Synthesized by the Pechini Process", J. Am. Ceram. Soc., 82 [7], 1681-1686 (1999).

5. D.M. Roy, S. O. Oyefesobi, "Preparation of very reactive $\mathrm{Ca}_{2} \mathrm{SiO}_{4}$ Powder", J. Am. Ceram. Soc., 60 [6], 178-180 (1977)
6. D.M. Roy, T.P. O'Holleran, R. R. Neurgaonkar, "Preparation and hydration studies of reactive $\beta-\mathrm{Ca}_{2} \mathrm{SiO}_{4}$ prepared by the EDS technique", Cement and Concrete Research, 75 [3], 337-342 (1978).

7. A.R. Ramachendran, M. W. Grutzeck, "Effect of $\mathrm{pH}$ on the hydration of tricalcium silicate", J. Am. Ceram. Soc., 76 [1], 72-80 (1993).

8. Q. Yu, K. Sawayama, S. Suyita, M. Shoya, Y. Isojima, "The reaction between rice huso ash and $\mathrm{Ca}(\mathrm{OH})_{2}$, solution and the nature of its product", Cement and Concrete Research, 29, 37-43 (1999).

9. M.H.Zhang, R. Latra, V. M, Malhotra, "Rice-Husk ash paste and concrete: Some aspects of hydration and the microstructure of the interfacial zone between the aggregate and paste", Cement and Concrete Research, 26 [6], 963-977 (1996).

10. M. Zhang, V. M. Malhotra, "High-performance concrete incorporating rice husk ash as a supplementary cementing material", ACI Materials Journal, November- December, 629-636, 1996.

11. J.E. Rodríguez-Páez., A.C. Caballero, M. Villegas, C. Moure, P. Durán, J.F. Fernández, "Controlled precipitation methods: formation mechanism of ZnO nanoparticles", J. Eur. Ceram. Soc., 21, 925-930 (2001).

12. A. Chakraverty, P. Mishra, H.D. Benerjee, "Ivestigation of combustión of raw and acid-leached rice husk for production of pure amorphous white silica", J.Mater. Sci., 23, 21-24 (1988)

13. J.E. Rodríguez-Páez, Estudio de los Mecanismos de formación de partículas de $\mathrm{ZnO}$ con diseño morfológico y dimensional obtenidas por el método de precipitación controlada, Tesis Doctoral Universidad Autónoma de Madrid, 1999.

14. G. Varona, G. Vivas, J.E. Rodríguez-Páez, "Silicón dióxido from rice husk: Chemical and thermic effects", Anais do $38^{\circ}$ Congreso Brasileiro de Cerámica, 18 al 21 de junio de 1994 Rio de Janeiro R.J. Brasil, 1994

15. N.N. Greenwood, A. Earnshaw, Chemistry of the Element, Butterworth/ Heineman Ltd., 1995.

16. F. Burriel Marti, F. Lucena Conde, S. Arribas Jimeno, J. Hernández Méndez Química Analítica Cualitativa, Editorial Paraninfo, 15º edición, 1994.

17. C.F., Baes Jr., R.E. Mesmer, The hydrolysis of cations, John Wiley \& Sons, Inc., 1976. 
18. K.J. Laidler/ J.H. Meiser, Physical Chemistry, Houghton Mifflin Company, 1999.

19. J. Israelachvili, Intermolecular and Surface forces, second edition Academic Press, 1995.

20. J. M. Rodríguez Medallo, R. Marín Galvín, Fisicoquímica de aguas, Edición Díaz de Santos S.A., 1999.
21. M. Szwarc, Ionic polymerization fundaments, Hanser/Gardner Publications, Inc., 1996

22. G. Socrates, Infrared Characteristic Group Frecuencies, John Wiley \& Sons, 1994.

Recibido: 17.09.05

Aceptado: 29.04.05 